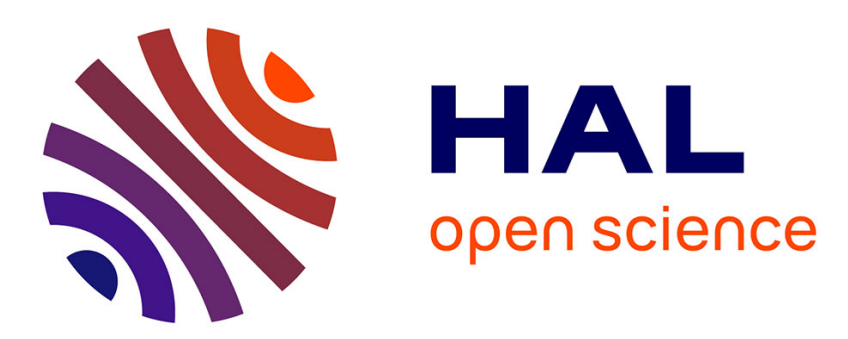

\title{
SPECTRAL-HOLE-BURNING-BASED OPTICAL ELEMENTS FOR TEMPORAL SHAPING OF LIGHT PULSES
}

\author{
H. Sónajalg, R. Kaarli, P. Saari
}

\section{- To cite this version:}

H. Sónajalg, R. Kaarli, P. Saari. SPECTRAL-HOLE-BURNING-BASED OPTICAL ELEMENTS FOR TEMPORAL SHAPING OF LIGHT PULSES. Journal de Physique IV Proceedings, 1991, 01 (C7), pp.C7-737-C7-739. 10.1051/jp4:19917198 . jpa-00250878

\section{HAL Id: jpa-00250878 https://hal.science/jpa-00250878}

Submitted on 1 Jan 1991

HAL is a multi-disciplinary open access archive for the deposit and dissemination of scientific research documents, whether they are published or not. The documents may come from teaching and research institutions in France or abroad, or from public or private research centers.
L'archive ouverte pluridisciplinaire HAL, est destinée au dépôt et à la diffusion de documents scientifiques de niveau recherche, publiés ou non, émanant des établissements d'enseignement et de recherche français ou étrangers, des laboratoires publics ou privés. 


\title{
SPECTRAL-HOLE-BURNING-BASED OPTICAL ELEMENTS FOR TEMPORAL SHAPING OF LIGHT PULSES
}

\author{
H. SÓNAJALG, R. KAARLI and P. SAARI \\ Institute of Physics, Estonian Academy of Sci, Riia 142, Tartu 202400, Estonia
}

\begin{abstract}
Two methods of optical pulse shaping and recalling are dlscussed. The pulse with arbltrary temporal modulations of the amplitude and the phase is formed due to free induction decay in anisotropic medium of a pllter.
\end{abstract}

\section{Introduction.}

Optical pulse shaping is of great interest in the study of light-matter interactions as well as in various applications. For this purpose it might be rather intriguing to make use of coherent transients, such as photon echo or free induction decay. In these phenomena a sample of a resonant medium acts on light as a complex array of long delay lines even though the sample is arbitrarily thin. In the pico- and femtosecond-scale $\left(10^{-12}-10^{-15} \mathrm{~s}\right)$ the employment of a spectral-hole-burning (SHB) /1/ material as the resonant medium seems to be prospective.

In ref./2/, the possibillty of shaping the temporal proflles of picosecond light pulses by filtration through fllters synthesized via SHB (SHB filters) was demonstrated experimentally. In these experiments, attention was paid to the scalar dielectric permittivity of the SHB material. In this case of isotropic spectral filters, the polarization state of a linearly polarized input pulse is preserved in the course of propagation. It was shown that the class of impulse responses of isotropic spectral filters is restricted. Namely, the impulse response of the isotropic filter $R_{0}(t)$ has to have the least first moment $\int t \cdot R(t) d t$ among the causal functions $R_{i}(t)$ with an identical power spectrum $T(\omega)$. The responses which satisfy this condition are referred to as the minimum-phase ones $13 /$. As a matter of pact, by making use of an elliptically polarized irradiation one may engineer a tensor of dielectric permittivity of an SHB material and synthesize anisotropic spectral elements. The anisotropy can be induced due to the fact that the dipole moments of the transition of photoactive impurity molecules are orientated at two or more selected directions as in crystals, or uniformly at all directions as in glassy materials.

In this paper two methods of linearly polarized optical pulse shaping based on anisotropic SHB elements (the filters and the holograms) are proposed.

\section{Anisotropic SHB filter.}

In this section we consider an optical scheme made up of a proper anisotropic SHB filter, which is placed between two crossed polarizers. Particularly, we propose a mathematical algorithm for preparing the anisotropic SHB fllter $/ 4 /$.

Let us Imagine a plate of an SHB material with the dipole moments of the transition of impurities orlentated at two perpendicular (say $x-$ and $y-$ ) directions. Before hole burning the optical properties of the plate for $x$-polarized probe are exactly equal to that of the $y$-polarized light. To prepare an anisotropic filter a process 
of twofold scanning of a monochromatic linearly ( $x-$ and $y-$ ) polarized irradiation is sultable. The pulse shaper consists of an input polarizer orientated at $45^{\circ}$ with $x-$ and $y$-axes, the anisotroplc filter and an output polarizer, which is crossed with respect to the input one. The spectral response of the pulse shaper is

$$
r(\omega)=\frac{1}{2}\left[r_{x}(\omega)-r_{y}(\omega)\right]
$$

where the responses of the filter for $x-$ and $y$-polarized probes, $r_{x}(\omega), r_{y}(\omega)$, are both the minimum-phase ones. Taking into account that the Fourier transform $r(\omega)$ of an arbitrary amplitude-phase modulated pulse $R(t)$ can be expressed as $r(\omega)=(1-i \not t) P(\omega)$ (the symbol $\not$ denotes the Hilbert transformation) and presenting the real part $f(\omega)$ of $r(\omega)$ as a difference of two non-negative functions $f_{j}(j=x, y)$, $f(\omega)=p_{x}(\omega)-p_{y}(\omega)$, one may obtain that:

an anisotropic filter with the transparency spectra

$$
\begin{aligned}
& T_{x}(\omega)=\left|(1-i \not z) f_{x}(\omega)\right|^{2} \\
& T_{y}(\omega)=\left|(1-i \not z) f_{y}(\omega)\right|^{2}
\end{aligned}
$$

for $x-$ and $y$-polarized probes, correspondingly, combined with two polarizers as described above, reshapes a $\delta$-like input pulse into an arbitrary response $R(t)$.

Note that the algorithm is realizable not only by making use of an anisotropic SHB filter but also by means of destructive interference of the responses of two isotropic spectral filters with the transparency spectra $T_{1}(\omega)=T_{x}(\omega)$ and $\mathrm{T}_{2}(\omega)=\mathrm{T}_{\mathrm{y}}(\omega)$, respectively.

Computer simulations are made to show the credibility of the proposed method. We take, for instance, an impulse response in the form of two temporally separated $\delta$-llke pulses: $R(t)=A \cdot \delta(t)+B \cdot \delta(t-\tau)$. If the second pulse is more intense compared with the front one (i.e. $B>A$ ), the response $R(t)$ has no minimum-phase. However, one can find two minimum-phase responses, $r_{x}(\omega)$ and $r_{y}(\omega)$, destructive interference of which gives the proper output $r(\omega)$ (figure 1 ).
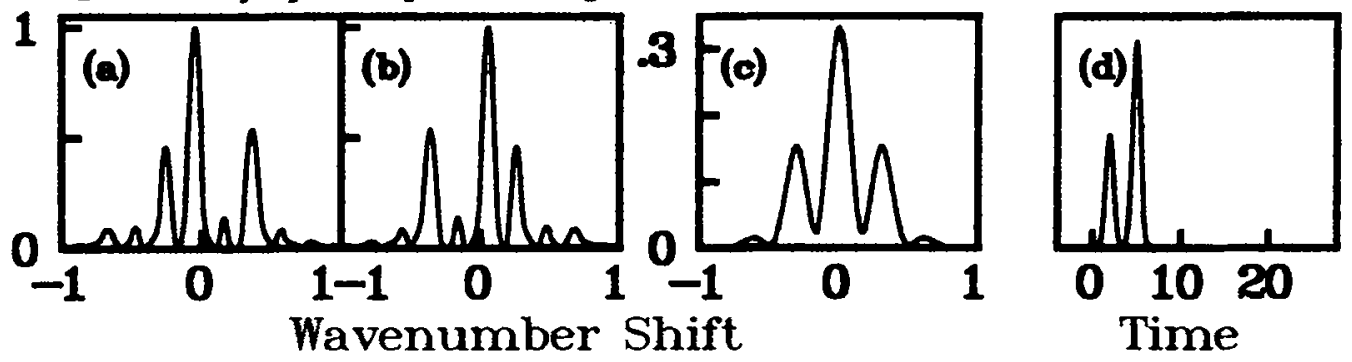

Fig.1. Computer simulation of the pulse shaping by making use of the anisotropic spectral filter. The spectra $\left|r_{x}(\omega)\right|^{2}$ and $\left|r_{y}(\omega)\right|^{2}$ of the two computed minimum-phase responses $(a, b)$, the transparency spectrum of the pulse shaper $|r(\omega)|^{2}$ (c), and the amplitude of the impulse response of the pulse shaper (d).

\section{Anisotropic SHB hologram of a linearly polarized object pulse.}

In this section we discuss a scheme of recording and recalling of an anisotropic spectral hologram. The advantages of this scheme of the holography are the following: (1) the object pulse can be recalled without the typical non-diffracted part of the impulse response of the spectral hologram, and, (ii) due to collinearity of the scheme, the reconstruction of the hologram is not too sensitive to the wavefront and to the direction of propagation of the read-out pulse.

To record the hologram an SHB plate is illuminated by the circularly polarized reference pulse $R_{c}(t)$ and the object pulse $S(t)$. Before reaching the plate, the 
linear polarization of the object pulse is converted into the circular one with the fleld vector rotating in the direction opposite to that of the reference pulse:

$$
\begin{aligned}
& \vec{R}_{c}(t)=\left(\vec{e}_{x}+i \vec{e}_{y}\right) \delta(t) \\
& \vec{S}_{c}(t)=\left(\vec{e}_{x}-i \vec{e}_{y}\right) S(t) .
\end{aligned}
$$

Here $\vec{e}_{x}, \vec{e}_{y}$ are the unit vectors along the $x$ - and $y$-axes, respectively, assuming the beams propagate along the $z$-axe. The response of the hologram to a $\delta$-like linearly polarized read-out pulse consists of two linearly polarized pulses whose pield vectors are, correspondingly, in the polarization plane of the read-out puise and in the perpendicular plane. The former is a non-diffracted part of the response and can be blocked out by the output polarizer, whereas the latter is the replica of the object signal.

To show that we once again consider the case of orthogonal orientations of the photoactive impurity molecules. The response of the anisotropic hologram combined with two crossed polarizers is given by eq.(1), where $r_{x}(\omega), r_{y}(\omega)$ are the responses of the two holograms burnt in with the $x$ - and $y$-components of the reference and signal pulses, correspondingly. Following the theory of space-and-time domain holography in spectrally selective photochromic materials $15-7 /$, one gets for the spectral responses of the two holograms:

$$
\begin{aligned}
& r_{x}(\omega)=r_{0}+x(1-i x)\left[|1(\omega)+s(\omega)|^{2}\right] \\
& r_{y}(\omega)=r_{0}+x(1-i x)\left[|1(\omega)-s(\omega)|^{2}\right] .
\end{aligned}
$$

Here $r_{0}$ stands for the response of a non-exposed plate, and $x$ depends on the parameters of the SHB material and on the recording doses. The corresponding impulse responses are

$$
\begin{aligned}
& R_{x}(t)=r_{0} \cdot \delta(t)+2 x \cdot Y(t)[\delta(t)+S(t)=S(t)+S(t)+S(-t)] \\
& R_{y}(t)=r_{0} \cdot \delta(t)+2 x \cdot Y(t)[\delta(t)+S(t)=S(t)-S(t)-S(-t)],
\end{aligned}
$$

and the response of the plate placed between crossed polarizers is (see eq.(1))

$$
R(t)=2 x \cdot Y(t)[S(t)+S(-t)] \text {. }
$$

In these equations $Y(t)$ denotes the unit step function and the symbol * indicates the operator of autocorrelation.

As one can see from eq.(6), the hologram reconstructs the object signal in its original form or with reversed time behaviour depending on the temporal order of the reference and the object pulses in the course of recording the hologram.

\section{Conclusion.}

The theory developed and the pulse shaping methods proposed are useful for the study of coherent optical transients and for various applications.

\section{References.}

/1/ Zero-phonon lines and spectral hole burning in spectroscopy and photochemistry, eds. O.Sild and K.Haller (Springer, Berlin, 1988).

/2/ H.Sónajalg, A.Gorokhovskit, R.Kaarli, V.Palm. M.Rätsep and P.Saari, Optlcs Comm. 71 (1989) 377.

/3/ J.S.Toll, Phys. Rev. 104 (1956) 1760.

/4/ H.Sónajalg. Optics Comm. 80 (1990) 76.

/5/ A.Rebane, R.Kaarli, P.Saari, A.Anijalg and K.Timpmann, Optics Comm.47 (1983)173.

/6/ P.Saarl, R.Kaarll and A.Rebane, J. Opt. Soc. Am. B 3 (1986) 527.

/7/ P.Saari, in reP./1/, p.123. 